'Departamento de Nutrición y Dietética, Facultad de Farmacia, Universidad de Concepción. Concepción, Chile.

2Programa de Magíster en Nutrición

Humana, Facultad de Farmacia, Universidad de Concepción. Concepción, Chile. ${ }^{3}$ Instituto de Farmacia, Facultad de Ciencias, Universidad Austral de Chile, Valdivia, Chile. ${ }^{4}$ Instituto de Anatomía, Histología y Patología, Facultad de Medicina, Universidad Austral de Chile. Valdivia, Chile. ${ }^{5}$ CIEDE-UCSC, Departamento de Salud Pública, Facultad de Medicina, Universidad Católica de la Santísima Concepción. Concepción, Chile.

${ }^{6}$ Escuela de Nutrición y Dietética, Facultad de Salud, Universidad Santo Tomás, Chile.

${ }^{7}$ Departamento de Psicología y Centro de Vida Saludable. Facultad de Ciencias Sociales. Universidad de Concepción. Concepción, Chile. 8BHF Glasgow Cardiovascular Research Centre, Institute of Cardiovascular and Medical Sciences, University of Glasgow. Glasgow, United Kingdom

${ }^{9}$ Centro de Investigación en Fisiología del Ejercicio-CIFE, Universidad Mayor. Santiago, Chile.

${ }^{10}$ Institute of Health and Wellbeing, University of Glasgow. Glasgow, United Kingdom. aBioquímico. ${ }^{b}$ Nutricionista. 'Profesora de Biología y Química. dPsicólogo. eProfesor de Educación Física. ${ }^{\mathrm{f}} \mathrm{PhD}$ ${ }^{9} \mathrm{MSC}$

*MM, KRA y AML contribuyeron de igual forma a este manuscrito y son considerados primer autor compartido.

Trabajo no recibio financiamiento Los autores declaran no tener conflictos de interés

Recibido el 28 de noviembre de 2019 aceptado el 9 de enero de 2020

Correspondencia a: Fanny Petermann-Rocha

BHF Glasgow Cardiovascular Research Centre Institute of Cardiovascular and Medical Sciences. College of Medical, Veterinary and Life Sciences. University of Glasgow.

Glasgow, G12 8TA. United Kingdom f.petermann-rocha.1@research.gla.ac.uk

\section{Menopausia y factores de riesgo cardiovascular en mujeres chilenas}

\author{
MIQUEL MARTORELL ${ }^{1, a, f, *,}$ \\ KARINA RAMÍREZ-ALARCÓN ${ }^{1 \mathrm{~b}, \mathrm{~g}, *}$, \\ ANA MARÍA LABRAÑA ${ }^{1, \mathrm{bg}, *, *}$, DANAHE BARRIENTOS ${ }^{2, \mathrm{~b}}$, \\ MAKARENA OPAZO ${ }^{2, b}$, \\ MARÍA ADELA MARTÍNEZ-SANGUINETTI ${ }^{3, a, g,}$ \\ ANA MARÍA LEIVA ${ }^{4, c, g,}$, CLAUDIA TRONCOSO-PANTOJA \\ NICOLE LASSERRE-LASO ${ }^{6, \mathrm{~b}, \mathrm{~g}}$, GABRIELA NAZAR R, ${ }^{7, \mathrm{~d}, \mathrm{f}}$ \\ CARLOS CELIS-MORALES ${ }^{8,9,10, e, f}$, \\ FANNY PETERMANN-ROCHA ${ }^{8,10, \mathrm{~b}, \mathrm{~g}}$ \\ (en representación del grupo de investigación ELHOC)
}

\section{Association of menopause with cardiovascular risk. Analysis of data from the 2016-17 Chilean national health survey}

Background: Menopause exposes women to an increased cardiovascular risk. Aim: To determine the association between menopause and cardiovascular risk factors in Chilean adult women using data from the National Health Survey (NHS) 2016-2017. Material and Methods: Data from 2,139 women over 40 years of age participating in the NHS 2016-2017, with information about menopause and cardiovascular risk factors was used. Expansion factors were applied to this sample, obtaining an expanded sample of 3,733,191 participants. Laboratory values (blood glucose, triglycerides, HDL and total cholesterol) and anthropometric measurements (body weight, height and waist circumference) were analyzed. The presence of hypertension, diabetes, and metabolic syndrome (MetS) were also recorded. Results: Sixty seven percent of surveyed women were menopausal and had higher systolic blood pressure than non-menopausal participants. Menopause was significantly associated with hypertension (Odds ratio (OR): 2.43 [95\% confidence intervals $(C I): 1.71 ; 3.45], p<0.01)$ and diabetes (OR: 2.05 [95\% CI: 1.32; 3.19], $p<0.01$ ). However, no association was observed with obesity, abdominal obesity or MetS. Conclusions: In these women, a positive association was identified between menopause and hypertension as well as diabetes.

(Rev Med Chile 2020; 148: 178-186)

Key words: Cardiovascular Diseases; Diabetes Mellitus; Hypertension; Menopause; Obesity. 


\section{I} a Organización Mundial de la Salud (OMS) define menopausia como el cese permanente $\checkmark$ de la menstruación espontánea tras un período de doce meses ${ }^{1}$. La mayoría de las mujeres comienzan este proceso entre los 49 y 52 años aproximadamente y se correlaciona, principalmente, con el cese de producción de ovocitos y cambios en su ciclo menstrual2,3. En este contexto, y considerando que la esperanza de vida de las mujeres supera los 80 años en muchos países ${ }^{1}$, la mayoría de ellas vive más de un tercio de sus vidas en un estado postmenopáusico, con las implicancias en su salud que esto conlleva ${ }^{4}$. En Chile, el Censo 2017 identifica que 51\% de la población es de sexo femenino ${ }^{5}$, según la Encuesta Nacional de Salud 2016-2017 (ENS 2016-2017), aproximadamente 1,3 millones de mujeres entre 45 y 64 años cursan con menopausia 6 . Además, se estima que para el año 2050, la esperanza de vida de las mujeres sea de 87,8 años, representando la población mayor de 65 años el 25\% total de la población. Esto significa que el número de mujeres en esta etapa aumentará exponencialmente ${ }^{5}$.

La menopausia provoca una modificación en la composición corporal, con pérdida de masa magra y aumento de masa grasa, junto con cambios hormonales, que incluyen la reducción drástica de hormonas ováricas ${ }^{7-10}$. Además, se produce una redistribución de la grasa corporal caracterizada por una reducción de la grasa subcutánea e incremento de la grasa visceral intraabdominal ${ }^{11}$. El mecanismo que relaciona la obesidad abdominal con enfermedades metabólicas presentes en esta etapa del ciclo vital estaría determinado por la alteración en la secreción de las adipocinas, gatillando una inflamación crónica ${ }^{12}$. Además, se observa una reducción de los niveles de hormonas femeninas, especialmente el estrógeno $y$, en consecuencia, un aumento en el riesgo de enfermedades cardiovasculares $(\mathrm{ECV})^{13}$. A nivel mundial, se estima que 8,6 millones de mujeres mueren cada año por ECV, lo que representa un tercio de todas las muertes en este grupo de la población ${ }^{4,14}$. El aumento de la esperanza de vida implica un aumento del número absoluto de las muertes por ECV en las mujeres ${ }^{4,14}$, lo que significa que la menopausia podría considerarse como un factor de riesgo de $\mathrm{ECV}^{15}$.

La menopausia, como sus manifestaciones metabólicas, puede aumentar el riesgo de presentar estrés oxidativo, aumentando el riesgo de desarrollar estados inflamatorios y enfermedades crónicas tales como: síndrome metabólico (SM), hipertensión arterial (HTA), diabetes mellitus tipo 2 (DM2), enfermedad renal crónica, ECV y dislipidemia. Sin embargo, es más probable que el aumento del estado oxidativo de la menopausia se deba a los mayores niveles de adiposidad en mujeres con esta condición y no a la menopausia por si sola ${ }^{16,17}$. Considerando el actual perfil epidemiológico chileno, donde se estima que hay 2.112.987 de mujeres con menopausia, y que este número aumentará debido al envejecimiento de la población, lo cual podría tener importantes repercusiones en el aumento de ECV, el objetivo de este estudio fue determinar la asociación entre menopausia y factores de riesgo cardiovascular en mujeres adultas chilenas utilizando datos de la ENS 2016-2017.

\section{Material y Métodos}

\section{Diseño del estudio}

Investigación basada en los datos de la ENS 2016-2017. La ENS 2016-2017 es un estudio de prevalencia realizado en hogares en una muestra nacional tipo transversal aleatoria, estratificada por conglomerado de 6.233 personas de 15 años o más, chilenas o extranjeras que residían habitualmente en viviendas particulares ocupadas, localizadas en zonas urbanas y rurales de las quince regiones de Chile. El estudio incluyó una muestra total de 2.139 mujeres mayores de 40 años, que tenían información disponible en relación a menopausia y a las variables de este estudio. Las preguntas específicas de la ENS 2016-2017 para determinar menopausia fueron: “ $¿$ cuándo fue su última menstruación o regla?”, “¿qué edad tenía cuando se le terminó/cortó definitivamente la menstruación o regla?”, y “ipodría indicarme aproximadamente entre qué edades se le terminó/ cortó la menstruación o regla?". Para ponderar la muestra a población nacional, se aplicaron los factores de expansión sugeridos por la ENS 20162017. Por lo tanto, la muestra expandida incluida en este estudio representa a 3.733.191 mujeres mayores de 40 años. El protocolo de la ENS fue aprobado por el Comité de Ética de la Escuela de Medicina de la Pontificia Universidad Católica de Chile y todos los participantes firmaron un consentimiento informado. 


\section{Mediciones metabólicas y antropométricas}

Los parámetros metabólicos, tales como glicemia basal, hemoglobina glicosilada, colesterol total, HDL y triglicéridos (TG), fueron medidos con ayuno $\geq 8 \mathrm{~h}$, a través de punción venosa, con métodos estandarizados y previamente descritos en la ENS 2016-2017 ${ }^{18}$. La sospecha y detección de HTA, se recopiló a través de autorreporte, información de tratamiento médico HTA o con presión arterial elevada $\geq 140 / 90 \mathrm{mmHg}$ (promedio de 3 tomas de presión arterial) medida a través de monitor de presión arterial HEM 7200. Para la DM2, se consideró una glicemia en ayuno $\geq 126 \mathrm{mg} / \mathrm{dL}$ o el autorreporte de diagnóstico médico de DM2. Para la variable SM, se consideró la presencia de tres o más de los siguientes cinco criterios definidos en ATP III Update 2004 ${ }^{19}$ : colesterol total elevado ( $\geq 200 \mathrm{mg} / \mathrm{dL})$, HDL bajo $(<50 \mathrm{mg} / \mathrm{dL}$ mujeres) y LDL elevado $(\geq 100 \mathrm{mg} /$ $\mathrm{dL})$, TG elevados $(\geq 150 \mathrm{mg} / \mathrm{dL})^{6}$.

La medición del peso y la talla corporal fueron recolectados mediante métodos estandarizados, tal como ha sido reportado anteriormente ${ }^{18,20}$. El estado nutricional se clasificó según índice de masa corporal (IMC) en base a las recomendaciones de la OMS para adultos ${ }^{21}$, mientras que la obesidad central fue definida por circunferencia cintura (CC) $\geq 88 \mathrm{~cm}$ para mujeres ${ }^{6}$.

\section{Variables sociodemográficas y de estilos de vida}

Las variables sociodemográficas consideradas para el estudio fueron: edad, sexo, zona geográfica, nivel educacional e ingreso económico, las cuales fueron recolectadas a través de cuestionarios validados en la ENS 2016-2017 . Los datos asociados con estilos de vida fueron: autorreporte de salud y bienestar, horas de sueño al día, hábito tabáquico, consumo diario de agua y cumplimiento de las Guías Alimentarias Basadas en Alimentos (GABA), para la población chilena ${ }^{22,23}$. El consumo de sal se determinó mediante análisis de excreción de sodio en orina de 24 h, mediante la ecuación de Tanaka ${ }^{24}$.

La actividad física (AF) total fue expresa en $\mathrm{MET} / \mathrm{min} /$ día y evaluada en 3 dimensiones, incluyendo la suma del tiempo reportado en actividades de transporte, en el trabajo y tiempo libre. Los niveles de AF y su intensidad (ligera, moderada o vigorosa) fueron determinados con el cuestionario Global Physical Activity Questionnaire (GPAQ v2) ${ }^{25}$.

\section{Análisis estadístico}

Los datos de caracterización de la población estudiada son presentados como promedios para variables continuas o como proporción para variables categóricas, con su respectivos 95\% intervalo de confianza (95\% IC) para mujeres con y sin diagnóstico de menopausia.

Diferencias entre las variables antropométricas (peso corporal, IMC y CC) y metabólicas (PAS, PAD, glicemia, colesterol total, TG, HDL, LDL) estudiadas entre personas con y sin menopausia, fueron investigadas mediante análisis de regresión lineal. Estos análisis fueron ajustados por las variables: nivel educacional, hábito tabáquico y nivel de AF. En aquellos marcadores donde adiposidad no fue la variable de salida, los análisis fueron ajustados, además, por IMC.

La asociación entre menopausia y las variables obesidad, obesidad central, DM2, SM y sospecha de HTA, fueron investigadas mediante regresión logística y reportada como odds ratio (OR) con sus respectivos 95\% IC. Estos análisis fueron ajustados por variables de confusión mediante la utilización de tres modelos estadísticos. Modelo 1 - no ajustado; Modelo 2 -ajustado por nivel educacional y zona de residencia (rural/urbana) y Modelo 3 - ajustado por el Modelo 2, pero también por AF y hábito tabáquico. Además, para evaluar si la adiposidad podría ser un mediador en la asociación entre menopausia con las variables DM2, SM y sospecha de HTA, estas fueron, además, ajustadas por IMC (Modelo 4) y CC (Modelo 5).

Para todos los análisis se utilizó el programa STATA MP v14 y el comando de "svyset" para muestras complejas. Todos los resultados fueron estimados utilizando muestras expandidas acorde a los factores de expansión sugeridos por la ENS 2016-2017. El nivel de significancia fue definido como $\mathrm{p}<0,05$.

\section{Resultados}

Las características de las participantes con y sin menopausa se identifican en la Tabla 1. En general, se destaca que $67 \%$ de las participantes reportaron menopausia y fueron en promedio 14,7 años mayores que las sin menopausia.

En la Figura 1 se observan las principales diferencias en marcadores antropométricos en mujeres con y sin menupausia. Estos análisis fue- 
Tabla 1. Características de las participantes, sin y con menopausia Update on selected markers used in risk assessment for vascular disease

\begin{tabular}{|c|c|c|c|c|}
\hline & \multicolumn{2}{|c|}{ Sin menopausia } & \multicolumn{2}{|c|}{ Con menopausia } \\
\hline n (encuestado)* & \multicolumn{2}{|c|}{705} & \multicolumn{2}{|c|}{1.434} \\
\hline n (muestra expandida)* & \multicolumn{2}{|c|}{1.620 .204} & \multicolumn{2}{|c|}{2.112 .987} \\
\hline Edad (años) & 48,5 & $(47,5 ; 49,6)$ & 63,2 & $(62,3 ; 64,1)$ \\
\hline Talla (m) & 1,56 & $(1,55 ; 1,56)$ & 1,53 & $(1,52 ; 1,54)$ \\
\hline $\begin{array}{l}\text { Estado nutricional (\%) } \\
\text { Bajo peso } \\
\text { Normopeso } \\
\text { Sobrepeso } \\
\text { Obeso }\end{array}$ & $\begin{array}{c}0,6 \\
16,8 \\
37,9 \\
44,6\end{array}$ & $\begin{array}{c}(0,1 ; 3,2) \\
(12,2 ; 22,8) \\
(31,5 ; 44,7) \\
(38,0 ; 51,1)\end{array}$ & $\begin{array}{c}4,2 \\
21,4 \\
35,3 \\
39,0\end{array}$ & $\begin{array}{c}(2,9 ; 6,0) \\
(17,8 ; 25,6) \\
(30,8 ; 40,1) \\
(34,4 ; 43,9)\end{array}$ \\
\hline Obesidad central, CC $\geq 88 \mathrm{~cm}(\%)$ & 71,7 & $(65,2 ; 77,3)$ & 72,4 & $(68,1 ; 76,3)$ \\
\hline Actividad física total (MET/min/día) & 960 & $(800 ; 1.120)$ & 650 & $(550 ; 749)$ \\
\hline Actividad física moderada (MET/min/día) & 253 & $(216 ; 289)$ & 195 & $(159 ; 231)$ \\
\hline Actividad física vigorosa (MET/min/día) & 212 & $(161 ; 262)$ & 188 & $(132 ; 244)$ \\
\hline Tiempo sedente (h/día) & 2,66 & $(2,32 ; 3,00)$ & 2,84 & $(2,57 ; 3,10)$ \\
\hline $\begin{array}{l}\text { Actividad física (\%) } \\
\text { Inactivo } \\
\text { Activo }\end{array}$ & $\begin{array}{l}29,5 \\
70,5\end{array}$ & $\begin{array}{l}(24,0 ; 35,6) \\
(64,4 ; 76,0)\end{array}$ & $\begin{array}{l}35,9 \\
64,1\end{array}$ & $\begin{array}{l}(31,4 ; 40,7) \\
(59,3 ; 68,6)\end{array}$ \\
\hline $\begin{array}{l}\text { Duración del sueño (\%) } \\
\text { Bajo, < } 7 \text { h/día } \\
\text { Normal, } 7-8 \text { h/día } \\
\text { Alto, }>8 \text { h/día }\end{array}$ & $\begin{array}{l}7,8 \\
20,6 \\
49,5 \\
29,9\end{array}$ & $\begin{array}{c}(7,6 ; 8,0) \\
(15,5 ; 26,8) \\
(42,8 ; 56,3) \\
(23,8 ; 36,8)\end{array}$ & $\begin{array}{c}7,2 \\
30,9 \\
50,4 \\
18,6\end{array}$ & $\begin{array}{c}(7,1 ; 7,4) \\
(26,3 ; 35,9) \\
(45,7 ; 55,3) \\
(15,5 ; 22,2)\end{array}$ \\
\hline $\begin{array}{l}\text { Nivel educacional (\%) } \\
\text { Básico, < } 8 \text { años } \\
\text { Medio, } 9-12 \text { años } \\
\text { Superior, }>12 \text { años }\end{array}$ & $\begin{array}{l}10,5 \\
19,9 \\
58,8 \\
21,3\end{array}$ & $\begin{array}{l}(10,1 ; 11,0) \\
(15,2 ; 25,5) \\
(51,9 ; 65,5) \\
(15,7 ; 28,2)\end{array}$ & $\begin{array}{l}8,67 \\
38,7 \\
49,3 \\
12,0\end{array}$ & $\begin{array}{r}(8,28 ; 9,04) \\
(34,4 ; 43,3) \\
(44,4 ; 54,1) \\
(9,2 ; 15,6)\end{array}$ \\
\hline $\begin{array}{l}\text { Nivel socio-económico (\%) } \\
\text { Bajo } \\
\text { Medio } \\
\text { Alto }\end{array}$ & $\begin{array}{l}33,9 \\
30,3 \\
35,8\end{array}$ & $\begin{array}{l}(27,5 ; 40,9) \\
(23,9 ; 37,6) \\
(28,6 ; 43,7)\end{array}$ & $\begin{array}{l}42,5 \\
34,8 \\
22,7\end{array}$ & $\begin{array}{l}(37,7 ; 47,4) \\
(29,7 ; 40,2) \\
(18,2 ; 28,0)\end{array}$ \\
\hline $\begin{array}{l}\text { Zona geográfica (\%) } \\
\text { Rural } \\
\text { Urbano }\end{array}$ & $\begin{array}{l}12,3 \\
87,7\end{array}$ & $\begin{array}{c}(9,7 ; 15,6) \\
(84,4 ; 90,3)\end{array}$ & $\begin{array}{l}12,1 \\
87,9\end{array}$ & $\begin{array}{l}(10,2 ; 14,3) \\
(85,7 ; 89,8)\end{array}$ \\
\hline $\begin{array}{l}\text { Autorreporte de salud y bienestar (\%) } \\
\text { Mala } \\
\text { Regular } \\
\text { Buena }\end{array}$ & $\begin{array}{l}3,8 \\
25,4 \\
70,8\end{array}$ & $\begin{array}{c}(1,5 ; 9,0) \\
(19,8 ; 31,9) \\
(63,9 ; 76,8)\end{array}$ & $\begin{array}{l}4,4 \\
29,0 \\
66,6\end{array}$ & $\begin{array}{c}(2,9 ; 6,7) \\
(24,9 ; 33,5) \\
(62,0 ; 70,9)\end{array}$ \\
\hline $\begin{array}{l}\text { Hábito tabáquico (\%) } \\
\text { Nunca ha fumado } \\
\text { Fumador ocasional } \\
\text { Fumador regular } \\
\text { Exfumadora }\end{array}$ & $\begin{array}{l}50,1 \\
6,9 \\
24,8 \\
18,2\end{array}$ & $\begin{array}{l}(43,3 ; 56,9) \\
(4,2 ; 11,4) \\
(19,1 ; 31,4) \\
(13,8 ; 23,5)\end{array}$ & $\begin{array}{c}48,3 \\
4,0 \\
18,0 \\
29,7\end{array}$ & $\begin{array}{l}(43,5 ; 53,1) \\
(2,5 ; 6,6) \\
(14,5 ; 22,1) \\
(25,4 ; 34,4)\end{array}$ \\
\hline Consumo agua (L/día) & 3,84 & $(3,46 ; 4,23)$ & 3,80 & $(3,55 ; 4,04)$ \\
\hline Consumo sal (g/día) & 9,18 & $(8,87 ; 9,48)$ & 9,45 & $(9,20 ; 9,71)$ \\
\hline $\begin{array}{l}\text { Cumplimiento consumo } 5 \text { frutas y verdur } \\
\text { Cumple } \\
\text { No cumple }\end{array}$ & $\begin{array}{l}11,2 \\
88,8\end{array}$ & $\begin{array}{c}(7,5 ; 16,2) \\
(83,8 ; 92,5)\end{array}$ & $\begin{array}{l}17,9 \\
82,1\end{array}$ & $\begin{array}{l}(14,6 ; 21,8) \\
(78,2 ; 85,4)\end{array}$ \\
\hline $\begin{array}{l}\text { Cumplimiento consumo } 3 \text { lácteos/día (\% } \\
\text { Cumple } \\
\text { No cumple }\end{array}$ & $\begin{array}{l}2,8 \\
97,2\end{array}$ & $\begin{array}{c}(1,6 ; 4,7) \\
(95,3 ; 98,4)\end{array}$ & $\begin{array}{l}5,2 \\
94,8\end{array}$ & $\begin{array}{c}(3,9 ; 7,0) \\
(93,0 ; 96,1)\end{array}$ \\
\hline $\begin{array}{l}\text { Cumplimiento legumbres } 2 \text { veces/seman } \\
\text { Cumple } \\
\text { No cumple }\end{array}$ & $\begin{array}{l}30,4 \\
69,6\end{array}$ & $\begin{array}{l}(24,2 ; 37,3) \\
(62,7 ; 75,8)\end{array}$ & $\begin{array}{l}25,4 \\
74,6\end{array}$ & $\begin{array}{l}(21,7 ; 29,4) \\
(70,6 ; 78,3)\end{array}$ \\
\hline
\end{tabular}

Datos presentados como media y su $95 \%$ IC para variables continuas y como porcentaje (\%) y su $95 \%$ IC para variables categóricas. Todos los valores fueron corregidos por el factor de expansión sugerido por la ENS 2016-2017. 
ron ajustados por las variables nivel educacional, hábito tabáquico y nivel de AF. Las mujeres con menopausia presentaron un menor peso corporal, IMC y CC $(69,6$ kg [95\% IC: 68,3; 70,1], 29,6 kg/ $\mathrm{m}^{2}$ [95\% IC: 29,$\left.1 ; 30,1\right], 94,4 \mathrm{~cm}$ [95\% IC: 93,0 ; $95,9])$, en comparación con las mujeres sin menopausia $(74,0 \mathrm{~kg}$ [95\% IC: 72,0; 75,9], 30,7 kg/ $\mathrm{m}^{2}$ [95\% IC: 29,$\left.9 ; 31,5\right], 96,5 \mathrm{~cm}$ [95\% IC: 94,7 ; $98,3])$.

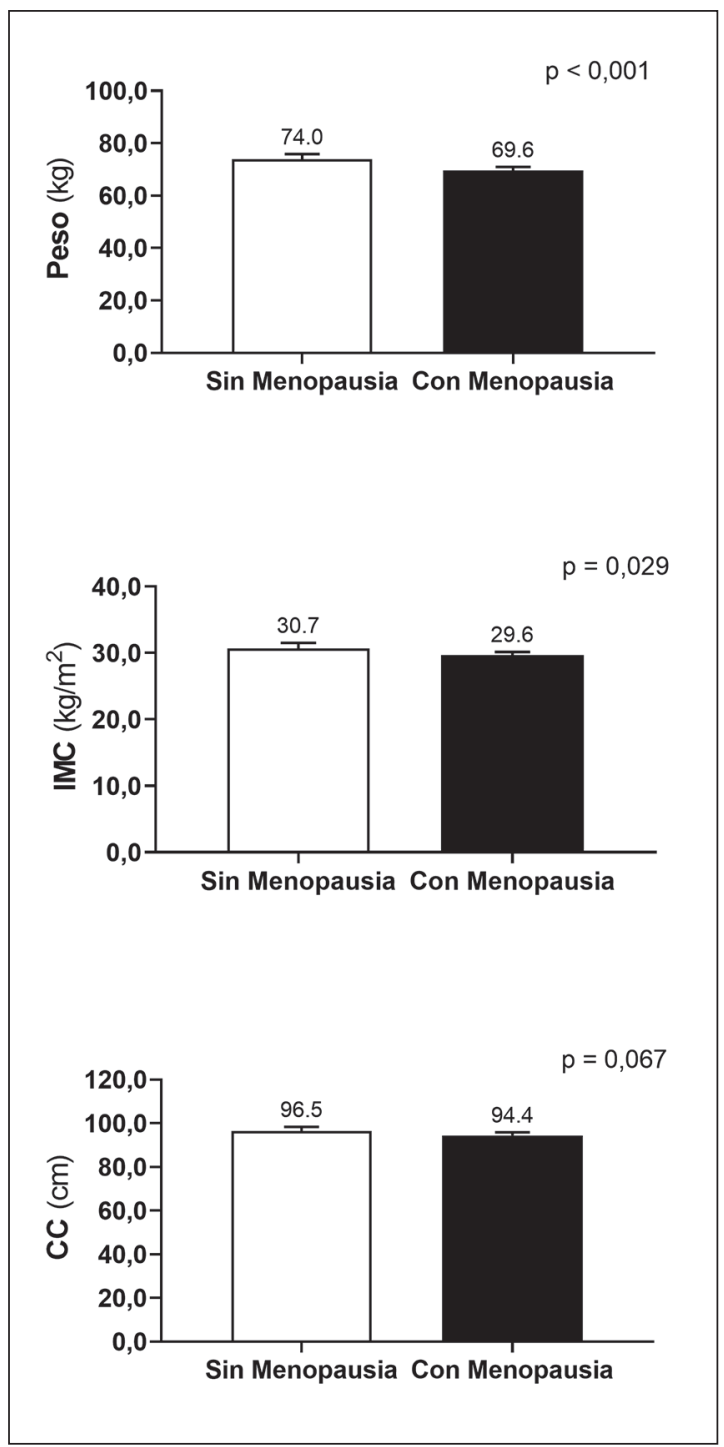

Figura 1. Asociación entre menopausia y marcadores antropométricos. Datos presentados como media y su respectivo 95\% IC. Estos análisis fueron ajustados por las variables nivel educacional, hábito tabáquico y nivel de actividad física.
En la Figura 2 se muestran las diferencias en marcadores metabólicos en mujeres con y sin menopausia. Los análisis fueron ajustados por variables de nivel educacional, hábito tabáquico, nivel de AF e IMC. Mujeres con menopausia reportaron una mayor PAS $(9,1 \mathrm{mmHg}, \mathrm{p}<0,001)$ y niveles de HDL $(2,5 \mathrm{mg} / \mathrm{dL}, \mathrm{p}=0,035)$. No se observaron diferencias significativas en PAD, glicemia, colesterol total, TG, ni LDL entre ambos grupos de mujeres (Figura 2).

La Tabla 2 muestra la asociación entre presencia de menopausia y variables antropométricas y metabólicas, según 5 modelos estádisticos mencionados en métodos. En relación a DM2, personas con menopausia presentaron una mayor probabilidad de desarrollarla en comparación a mujeres sin menopausia (Modelo 1, OR: 2,05 [95\%IC: 1,$32 ; 3,19]$ ), y a pesar de que al ajustar por otros modelos la asociación disminuyó, esta se mantuvo significativa. En el caso de HTA, las mujeres con menopausia presentaron 2,4 veces mayor probabilidad de sospecha de HTA (Modelo 1, OR: 2,43 [95\% IC: 1,71; 3,45]), esta asociación fue levemente modificada al ajustar por variables sociodemográficas como por estilos de vida (OR: 2,09 [95\% IC: 1,46; 3,00]). Al ajustar estos modelos estadísticos por IMC (OR: 2,37 [95\% IC:1,68; $3,36]$ ) o CC (OR: 2,44 [95\% IC: 1,72; 3,45]), estas asociaciones permanecieron significativas. No se identificaron asociaciones entre menopausia y obesidad, SM, u obesidad central, en ninguno de los modelos anteriormente señalados.

\section{Discusión}

La menopausia podría aumentar el riesgo de desarrollar ECV, por lo que es importante estudiar las alteraciones provocadas por la disminución de los niveles de estrógenos y cómo inciden en los factores de riesgo cardiovascular de las mujeres que presentan menopausia ${ }^{13,15}$. Los resultados del presente estudio muestran una asociación entre menopausia y los factores de riesgo cardiovascular, principalmente PAS aumentada, DM2 y sospecha de HTA. Además, los resultados muestran que las mujeres con menopausia presentan un menor peso corporal, IMC y CC, al compararlas con aquellas sin menopausia; no se identificaron asociaciones entre menopausia y obesidad u obesidad central (nivel de adiposidad) o SM, en ninguno 

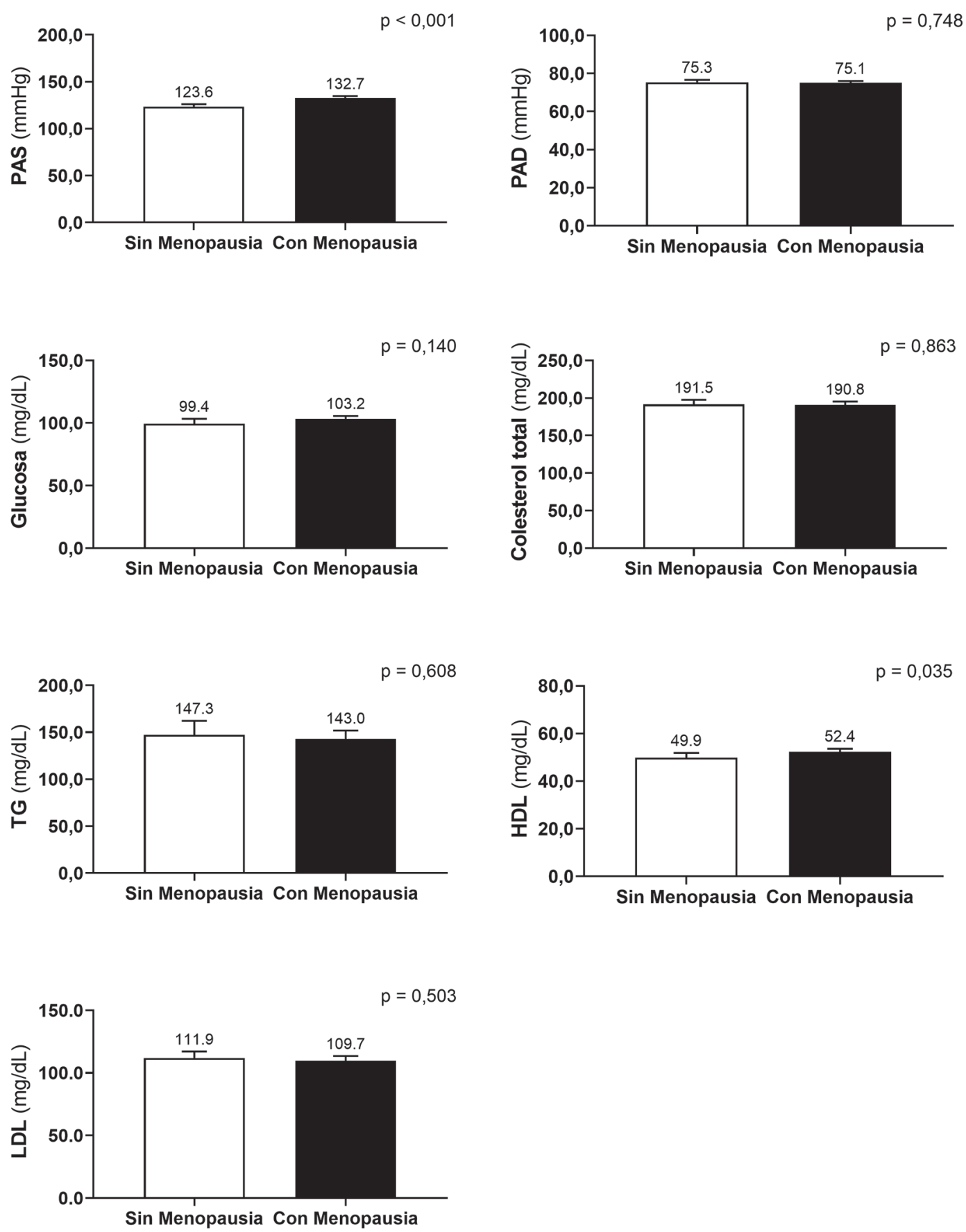

Figura 2. Asociación entre menopausia y marcadores metabólicos. Datos presentados como media y su respectivo $95 \%$ IC. Estos análisis fueron ajustados por las variables nivel educacional, hábito tabáquico y nivel de actividad física. En aquellos marcadores donde adiposidad no fue la variable de salida, los análisis fueron ajustados además por IMC. 


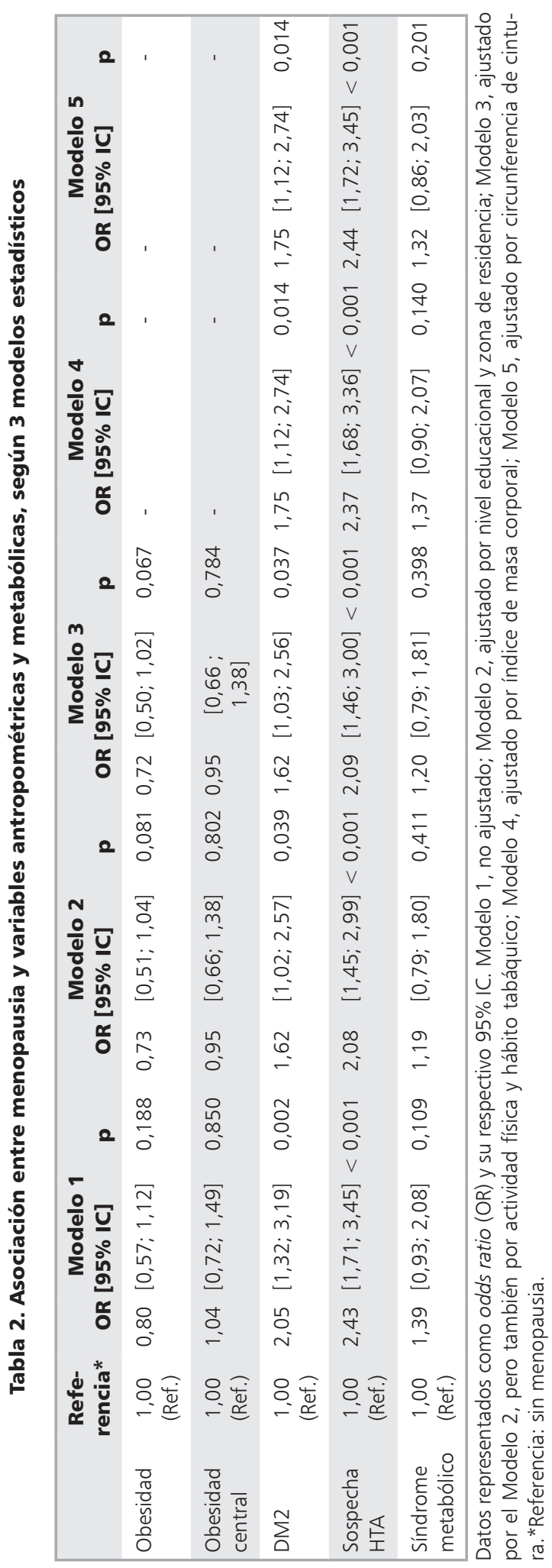

de los 5 modelos anteriormente señalados, lo que concuerda con lo reportado en otros trabajos internacionales realizados en esta misma población $^{26,27}$. Un estudio realizado en Estados Unidos de Norteamérica en 1.246 mujeres reportó resultados similares a los encontrados en este trabajo, señalando que la composición corporal y el peso corporal no aumentaron durante la postmenopausia ${ }^{26}$. Otro estudio prospectivo desarrollado en Taiwán observó que mujeres menopáusicas tuvieron una menor ganancia de peso corporal e $\mathrm{IMC}^{27}$. Sin embargo, existen otros trabajos cuyos resultados no coinciden con los de este estudio. Una investigación realizada en Estados Unidos de Norteamérica evidenció un aumento en la grasa intraabdominal en el estado postmenopáusico, al comparar 53 mujeres premenopáusicas versus 28 en etapa temprana de menopausia ${ }^{11}$. En la misma línea, una revisión del año 2016 señaló que la transición menopáusica se asocia con aumento de peso corporal y aumento de la grasa abdominal ${ }^{28}$.

Los resultados de este estudio señalan que la menopausia estaría relacionada con una mayor probabilidad de DM2 y sospecha de HTA, la cual se mantuvo significativa en todos los modelos estudiados, independiente de los factores de confusión utilizados. Resultados similares reportó un estudio realizado en China con más de 2.000 participantes, concluyendo que la menopausia puede aumentar el riesgo de presentar glicemia alterada en ayunas ${ }^{29}$. Un metaanálisis realizado por $\mathrm{Guo}^{30}$ señala que a mayor edad de inicio de la menopausia se asocia a un menor riesgo de $\mathrm{DM} 2$, indicando que con cada incremento de 5 años en la edad de inicio de la menopausia hay $10 \%$ menos de riesgo de esta enfermedad ${ }^{30}$. En cuanto a la sospecha de HTA, un estudio realizado en 13.406 mujeres chinas reportó que la edad de inicio temprano de la menopausia se asocia significativamente con un aumento de la prevalencia de $\mathrm{HTA}^{31}$. Otro estudio realizado en Argentina, en 1.034 mujeres con menopausia, observó una elevada prevalencia de HTA en esta población ${ }^{32}$.

En relación a menopausia y dislipidemia, en nuestro estudio, al ajustar por edad e IMC (Modelo 4), no se observaron diferencias significativas en los niveles de TG, LDL, ni colesterol total, solo hubo diferencias significativas en el HDL. Al respecto, resultados contradictorios han sido reportados en un estudio desarrollado en Argelia, que comparó un total de 210 mujeres sin menopausia 
con aquellas con menopausia ${ }^{17}$. Las mujeres con menopausia reportaron significativamente un menor nivel de HDL, sin embargo, este estudio también encontró que mujeres menopáusicas tenían un mayor peso corporal, IMC, CC, PAS, $\mathrm{PAD}$, colesterol total y $\mathrm{LDL}^{17}$.

\section{Fortalezas y limitaciones}

Una de las principales fortalezas del presente estudio es la utilización de datos de la ENS 20162017, que consiste en una encuesta que utilizó protocolos estandarizados, representativa de la población a nivel nacional. Además, según nuestro conocimiento, no existen estudios en población chilena que aborden la problemática de la menopausia y variables de riesgo cardiovascular en esta etapa del curso de vida de la mujer. Como limitaciones del estudio, se identifica el autorreporte de menopausia y algunos factores de riesgo cardiovascular. Además, si bien se aplicó un factor de expansión para expandir esta muestra a población nacional, esta es solo teórica. Finalmente, por tratarse de un estudio transversal, no permite extraer ninguna inferencia causal de los resultados.

\section{Conclusiones}

A la luz de los resultados, se concluye que existen asociaciones positivas entre la menopausia y factores de riesgo cardiovascular en mujeres chilenas, reportando mayores niveles de PAS, mayor probabilidad de tener DM2 e HTA, pero no con obesidad, obesidad abdominal o SM. Los hallazgos de este estudio apoyan la necesidad de realizar una mayor vigilancia de los marcadores metabólicos y antropométricos de las mujeres que están en este proceso fisiológico y la consecuente necesidad de reforzar los factores protectores. Por lo anterior, es necesario concientizar a las mujeres y a diversos actores del sistema de salud de la importancia de la promoción de los factores protectores y prevencion de los factores de riesgo cardiovascular durante el proceso de cambio fisiológico que ocurre durante la menopausia, mediante intervenciones a temprana edad.

Agradecimientos: Se agradece de manera especial a todos los participantes de la ENS 20162017, al equipo profesional de la Escuela de Salud
Pública, de la Facultad de Medicina de la Pontificia Universidad Católica de Chile, quienes desarrollaron y aplicaron la Encuesta Nacional de Salud y al Ministerio de Salud del Gobierno de Chile.

\section{Referencias}

1. WHO. Women, Ageing and Health: A Framework for Action. World Health Organization (WHO) Report. 2007.

2. Harlow SD, Gass M, Hall JE, Lobo R, Maki P, Rebar RW, et al. Executive summary of the Stages of Reproductive Aging Workshop + 10: addressing the unfinished agenda of staging reproductive aging. J Clin Endocrinol Metab 2012; 97 (4): 1159-68.

3. Morabia A, Costanza MC. International variability in ages at menarche, first livebirth, and menopause. World Health Organization Collaborative Study of Neoplasia and Steroid Contraceptives. Am J Epidemiol 1998; 148 (12): 1195-205.

4. Biglia N, Cagnacci A, Gambacciani M, Lello S, Maffei S, Nappi RE. Vasomotor symptoms in menopause: a biomarker of cardiovascular disease risk and other chronic diseases? Climacteric 2017; 20 (4): 306-12.

5. INE. CENSO 2017. Estimaciones y proyecciones de población. Instituto Nacional de Estadísticas (INE) de Chile. 2018; Disponible: https://www.censo2017.cl/.

6. MINSAL. Sabana datos oficial Encuesta Nacional de Salud (ENS) 2016-2017. Ministerio de Salud (MINSAL) - Gobierno de Chile. 2017; Disponible: https://inta.cl/ wp-content/uploads/2018/01/ENS-2016-17_PRIMEROS-321\%20RESULTADOS-1.pdf.

7. Noh H, Lee H, Kim S, Joo J, Suh D, Kim K, et al. The Efficacy of Body Mass Index and Total Body Fat Percent in Diagnosis Obesity according to Menopausal Status. J Menopausal Med 2019; 25 (1): 55-62.

8. Leeners B, Geary N, Tobler PN, Asarian L. Ovarian hormones and obesity. Hum Reprod Update 2017; 23 (3): 300-21.

9. Al-Safi ZA, Polotsky AJ. Obesity and menopause. Best Pract Res Clin Obstet Gynaecol 2015; 29 (4): 548-53.

10. Lovejoy JC, Champagne CM, de Jonge L, Xie H, Smith SR. Increased visceral fat and decreased energy expenditure during the menopausal transition. Int J Obes (Lond) 2008; 32 (6): 949-58.

11. Toth MJ, Tchernof A, Sites CK, Poehlman ET. Effect of menopausal status on body composition and abdominal fat distribution. Int J Obes Relat Metab Disord 2000; 24 (2): 226-31.

12. Harwood HJ. The adipocyte as an endocrine organ in 
the regulation of metabolic homeostasis. Neuropharmacology 2012; 63 (1): 57-75.

13. Newson L. Menopause and cardiovascular disease. Post Reprod Health 2018; 24 (1): 44-9.

14. Mozaffarian D, Benjamin EJ, Go AS, Arnett DK, Blaha MJ, Cushman M, et al. Executive Summary: Heart Disease and Stroke Statistics--2016 Update: A Report From the American Heart Association. Circulation 2016; 133 (4): 447-54.

15. Rosano GMC, Vitale C, Marazzi G, Volterrani M. Menopause and cardiovascular disease: the evidence. Climacteric 2007; 10 (sup1): 19-24.

16. Fu Y, Yu Y, Wang S, Kanu JS, You Y, Liu Y, et al. Menopausal Age and Chronic Diseases in Elderly Women: A Cross-Sectional Study in Northeast China. Int J Environ Res Public Health 2016; 13 (10).

17. Taleb-Belkadi O, Chaib H, Zemour L, Fatah A, Chafi B, Mekki K. Lipid profile, inflammation, and oxidative status in peri- and postmenopausal women. Gynecol Endocrinol 2016; 32 (12): 982-5.

18. MINSAL. Manual de aplicación de cuestionario F2, Encuesta Nacional de Salud 2016-2017. Ministerio de Salud (MINSAL) - Gobierno de Chile. 2016.

19. Carreiro-Lewandowski E. Update on selected markers used in risk assessment for vascular disease. Clin Lab Sci 2004; 17 (1): 43-9.

20. Petermann-Rocha F, Rocha C, Martínez-Sanguinetti MA, Leiva AM, Troncoso-Pantoja C, Villagrán M, et al. ¿Existe asociación entre asma y adiposidad en la población adulta chilena? Rev Med Chile 2019; 147: 733-40.

21. WHO. Obesity: preventing and Managing the Global Epidemic. World Health Organization (WHO) Report. 2000.

22. MINSAL. Guías Alimentarias para la Población Chilena. Disponible: https://wwwminsalcl/portal/url/item/dde0bc471a56a001e040010165012224pdf. 2013.

23. Olivares S, Zacarías I, González CG, Villalobos E. Proceso de formulación y validación de las guías alimentarias para la población chilena. Rev Chilena Nutr
2013; 40: 262-8.

24. Tanaka T, Okamura T, Miura K, Kadowaki T, Ueshima $\mathrm{H}$, Nakagawa $\mathrm{H}$, et al. A simple method to estimate populational 24-h urinary sodium and potassium excretion using a casual urine specimen. J Hum Hypertens 2002; 16 (2): 97-103.

25. Armstrong T, Bull F. Development of the world health organization Global Physical Activity Questionnaire (GPAQ). J Public Health (Oxf) 2006; 14 (2): 66-70.

26. Greendale GA, Sternfeld B, Huang M, Han W, Karvonen-Gutiérrez $\mathrm{C}$, Ruppert $\mathrm{K}$, et al. Changes in body composition and weight during the menopause transition. JCI Insight 2019; 4 (5).

27. Torng PL, Su TC, Sung FC, Chien KL, Huang SC, Chow $\mathrm{SN}$, et al. Effects of menopause on intraindividual changes in serum lipids, blood pressure, and body weight-the Chin-Shan Community Cardiovascular Cohort study. Atherosclerosis 2002; 161 (2): 409-15.

28. Karvonen-Gutiérrez C, Kim C. Association of Mid-Life Changes in Body Size, Body Composition and Obesity Status with the Menopausal Transition. Healthcare (Basel) 2016; 4 (3).

29. Wen JB, Yang S, Zhao XH, Chen YC, Zhao HL, Chen $\mathrm{XT}$, et al. [Effect of menopausal status on onset of impaired fasting glucose and type 2 diabetes mellitus]. Zhonghua yu fang yi xue za zhi (Chinese Journal of Preventive Medicine) 2017; 51 (8): 728-33.

30. Guo C, Li Q, Tian G, Liu Y, Sun X, Yin Z, et al. Association of age at menopause and type 2 diabetes: A systematic review and dose-response meta-analysis of cohort studies. Prim Care Diabetes 2019.

31. Song L, Shen L, Li H, Liu B, Zheng X, Zhang L, et al. Age at natural menopause and hypertension among middle-aged and older Chinese women. JoJ Hypertens 2018; 36 (3): 594-600.

32. Zilberman JM, Cerezo GH, Del Sueldo M, Fernández-Pérez C, Martell-Claros N, Vicario A. Association Between Hypertension, Menopause, and Cognition in Women. J Clin Hypertens (Greenwich) 2015; 17 (12): 970-6. 